After death audit data was collected in each care home following the death of a resident at baseline (i.e. prior to the intervention), and once the intervention had started.

Components of the intervention were: support from a dedicated Community Palliative Care Clinical Nurse Specialist; identification of two palliative care leads in each care home; palliative care training; multidisciplinary palliative care review meetings, and support to use tools to help identify residents approaching end of life.

Baseline data relating to 77 residents was collected: 92\% of residents died in the care home; $64 \%$ had a record of anticipatory care planning; $19 \%$ died on an integrated care pathway and $55 \%$ had anticipatory medicines prescribed. The intervention is continuing. Data on outcomes during the intervention period will be compared against the baseline data.

Conclusion Our study explores the extent to which a community palliative care nurse specialist led model of care home support enables care home staff to improve the delivery of palliative care to their residents. The findings will inform the delivery of the intervention to 14 care homes in Phase 2. More generally, this project will guide the design of new palliative care interventions that will allow palliative care specialists to extend their influence to reach patients with frailty and dementia.

\title{
P 261 A COMMUNITY PALLIATIVE CARE NURSE SPECIALIST LED INTERVENTION TO IMPROVE PALLIATIVE CARE IN CARE HOMES: PRELIMINARY RESULTS
}

\author{
Anne M Finucane, ${ }^{1}$ Hilary Gardner, ${ }^{1}$ Barbara Stevenson, ${ }^{1}$ Lynn Muir, ${ }^{1}$ \\ Hilary Gibson, ${ }^{1}$ Liz Barker, ${ }^{1}$ Scott A. Murray2. 'Marie Curie Hospice Edinburgh, \\ Edinburgh, Scotland, UK; ${ }^{2}$ University of Edinburgh, Edinburgh, Scotland, UK
}

10.1136/bmjspcare-2014-000654.302

Background Approximately 20\% of people die in long term care settings. National and international policies call for care homes to provide reliably good end-of-life care. Specialist palliative care providers are well placed to support care homes to deliver palliative care to residents who would benefit.

Aim To improve the delivery of palliative care in care home using a community palliative care clinical nurse specialist (CNS) model of support.

Results Twenty-two South Edinburgh nursing care homes agreed to take part in this project. Eight took part in Phase 1. 